\title{
Determinación de Bifenilos policlorados (PCB's) en trabajadores de una empresa del sector eléctrico. Barranquilla - Atlántico, 2017
}

\author{
Determination of polychlorinated biphenyls (PCB's) in workers of a \\ company in the electricity sector. Barranquilla - Atlantic, 2017
}

\author{
Aracely García-Cuan'; Ana Medina-Buelvas²; Jaime Pinedo-Otálvaro'; Nivaldo Rosales-Hernández²; Aldo Molina-Solano²
}

Forma de citar: Garcia-Cuan A, Medina-Buelvas A, Pinedo-Otálvaro J, Rosales-Hernández N, Molina-Solano A. Determinación de Bifenilos policlorados (PCB's) en trabajadores de una empresa del sector eléctrico. Barranquilla - Atlántico, 2017. Rev Univ Ind Santander Salud. 2019; 51(1): 23-32. doi: http://dx.doi.org/10.18273/revsal.v51n1-2019003 @) (1)

\section{Resumen}

Introducción: Se determinó Bifenilos Policlorados (PCB's) en sangre y pruebas de función orgánica para evaluación de la salud en trabajadores de una empresa del sector eléctrico. Métodos: Fueron seleccionados 61 trabajadores de la empresa Electricaribe S.A de Barranquilla, 30 expuestos al PCB's y 31 no expuestos, mayores de 18 años, sin patología establecida y que firmaron el consentimiento informado. Se analizó: glicemia, perfil lipidico, creatinina, nitrógeno ureico (BUN), bilirrubinas, fosfatasa alcalina, transaminasas pirúvica y oxaloacética, gammaglutamiltranspeptidasa, $\mathrm{T}_{3}, \mathrm{~T}_{4}$, hormona estimulante de la tiroides (TSH). Para la detección de PCB's, se utilizó un cromatógrafo de gases HP 5890 series II (Hewlett Packard), detector de captura de electrones (EDC) y columna DB-5 (30m x $0.25 \mathrm{~mm} \times 0.25 \mu \mathrm{m})$. Los datos fueron procesados con el software Minitab 15, nivel de confianza $95 \%$. Resultados: Se encontró que el 100\% de trabajadores examinados presentaron cifras de PCB's inferiores al valor límite mínimo de detección del método cromatográfico utilizado: $4 \mu \mathrm{g} / \mathrm{L}$ de PCB's, y evaluaciones clínicas negativas para cualquier daño orgánico. Conclusiones: No se encontró diferencia significativa en los resultados obtenidos entre trabajadores expuestos y no expuestos al PCB's.

Palabras clave: Bifenilos policlorados; PCB's; Electricistas; Toxicidad.

\begin{abstract}
Introduction: Polychlorinated Biphenyls (PCB's) in blood and tests of organic function for evaluation of the health in workers of a company of the electrical sector were determined. Methods: We selected 61 company employees Electricaribe S.A. of Barranquilla, 30 exposed to PCB's and 31 unexposed, over 18 years without established pathology and that signed the informed consent. It was analyzed: glycemia, lipid profile, creatinine, blood urea nitrogen (BUN), bilirrubin, alkaline phosphatase, pyruvic and oxaloacetic transaminase, gamma-glutamyltranspeptidase, T3, T4, thyroid stimulating hormone (TSH). For detection of PCB's, a chromatograph HP 5890 series II gas (Hewlett

1. Universidad Libre Seccional Barranquilla. Atlántico, Colombia

2. Electricaribe S.A E.S.P. Barranquilla, Colombia

Correspondencia: Aracely García Cuan. Dirección: Universidad Libre Barranquilla, Km 7 Antigua Vía Puerto Colombia. Teléfono: +(575)

3851057 Correo electrónico: agarciac@unilibrebaq.edu.co
\end{abstract}


Packard), electron capture detector (EDC) and DB-5 column (30 $\mathrm{m}$ x $0.25 \mathrm{~mm} \times 0.25 \mu \mathrm{m}$ ) was used. The data were processed with Minitab 15 software, 95\% confidence level. Results: It was found that $100 \%$ of workers examined showed PCB's values lower than the minimum limit value of detection of the chromatographic method used: $4 \mu \mathrm{g} /$ L of PCB's, and negative clinical evaluations for any organic damage. Conclusions: No significant difference in the results between exposed and unexposed to the PCB's workers.

Keywords: Polychlorinated biphenyls; PCB's; Electricians; Toxicity.

\section{Introducción}

Los PCB's son un grupo de más de 200 compuestos químicos organoclorados sintéticos. Algunos PCB's integran una familia de compuestos relacionados química y estructuralmente llamado "dioxinas", constituida por para-dibenzo-dioxinas-cloradas (PCDD) y los dibenzo-furanos-policlorados (PCDF), con propiedades tóxicas parecidas ${ }^{1}$. Los PCB's se forman mediante la cloración de alguna de las diez diferentes posiciones del bifenilo (Figura 1). Se presentan, desde líquidos grasos hasta sólidos cerosos; tienen elevado punto de ebullición y propiedades de aislante eléctrico, son estables, no inflamables. Las principales aplicaciones son como refrigerantes y lubricantes en transformadores y otros equipos eléctricos ${ }^{2}$.

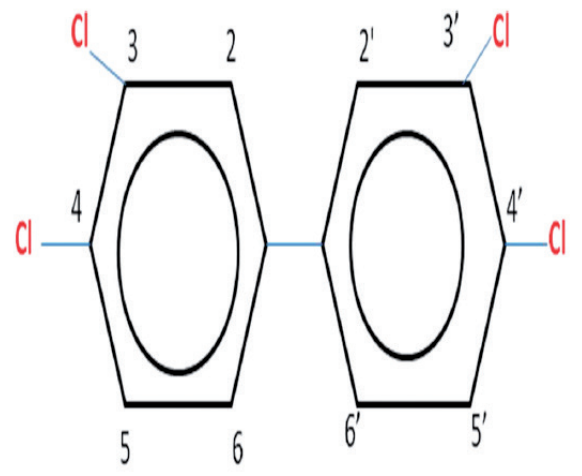

Figura 1. Derivado del bifenilo. Estructura del 3,3', 4,4' tetraclorobifenil (PBC-77)

Fuente: Elaborada por los autores.

En 1881, en Alemania, Schmitt-Schulz realizó la primera síntesis de PCB's3'; en 1927, Swann Chemical Company clasificaba sus PCB's como comerciales puros, conocidos como Arocloros, según su contenido de cloro. Así, Aroclor 1254 se refiere a PCB's con 54\% de cloro. Los Arocloros más utilizados como fluidos dieléctricos (Askarels) eran los Arocloros 1242,1254 y 1260. Desde 1929, Monsanto inicio su producción industrial y en 1935 absorbió a ésa empresa ${ }^{4}$. Actualmente, su uso está prohibido en casi todo el mundo y su fabricación, desde 1977 en Estados Unidos y 1983 en Alemania 5 .
Los PCB's, según el Programa de las Naciones Unidas para el Medio Ambiente (PNUMA), están incluidos entre los doce Contaminantes Orgánicos Persistentes (COP) más nocivos, los PCB's mantienen una persistencia alta en el ambiente y en el organismo humano (contacto accidental en la comunidad y profesional en trabajadores de la industria eléctrica), después de su emisión pueden permanecer desde tres semanas a dos años en el agua, y más de seis años en suelos y sedimentos, y 10 años en peces adultos contaminados con Bifenilos Policlorados, por lo tanto, son fuente de preocupación ambiental y de salud pública ${ }^{5,6}$. En 2001, más de 100 países firmaron la Convención de Estocolmo para eliminar el uso de éstos?.

En Colombia, en 1996 se aprobó el Convenio de Basilea sobre el control de movimientos fronterizos de desechos peligrosos ${ }^{8}$ y desde 1997, el tema de PCB's, es desarrollado con el proyecto CERI y la cooperación técnica del gobierno de Canadá9.

Los efectos tóxicos causados en el organismo se relacionan con exposición ocupacional y/o accidental; desde el punto de vista ocupacional, (la exposición puede ocurrir durante la reparación y mantenimiento de transformadores con PCB's, en Colombia todavía en algunas zonas se encuentran transformadores con PCB's que pueden estar en uso, en desecho o en almacenamiento y su vida útil es hasta 35 años) o por la ingestión de alimentos con residuos ${ }^{10}$. La Salud Ocupacional o la Seguridad y Salud en el Trabajo es un campo especializado de la práctica médica, compuesto por diferentes disciplinas que se basan en técnicas dirigidas a promover el cuidado de la salud y el bienestar de los trabajadores; también, impedir la presencia de la enfermedad, la incapacidad o la muerte prematura, consistiendo esta en una de las iniciativas de la sociedad para proteger, promover y recuperar la salud de la población, en otras palabras, la salud ocupacional hace parte de la Salud Pública, de manera que todas estas acciones que propenden por la salud de estos trabajadores están incidiendo en la salud de la población, ya que al mantener la salud de los trabajadores, mejoran o tienen una 
buena calidad de vida, que llega hasta sus familias y sociedad donde se desenvuelve el individuo, con repercusiones positivas para la sociedad, además de una excelente productividad para la empresa donde laboran.

Por su propiedad de asimilarse a los lípidos ("lipofilicidad") y su baja degradabilidad los PCB's tienden a acumularse a medida que avanzan en la cadena alimentaria; por esta razón, se encuentran en el tejido adiposo de animales terrestres, acuáticos y humanos, permaneciendo en ellos, con los consecuentes efectos toxicológicos ${ }^{11,12}$.

La exposición a niveles muy altos de PCB's por poco tiempo (por ejemplo, los trabajadores que desmantelan transformadores eléctricos, etc.) puede provocar despigmentación de la piel, brotes tipo acné, náuseas, vómitos, dolores de cabeza, mareos, adormecimiento y hormigueo en las pantorrillas, daño hepático ${ }^{13}$.

La exposición prolongada a los PCB's está vinculada con alteraciones inmunitarias, del sistema nervioso y endocrino, la función reproductora y la aparición de cáncer ${ }^{14,15}$. Varios estudios realizados en trabajadores expuestos reportan el aumento de diversos tipos de cáncer, como de hígado ${ }^{16}$, páncreas ${ }^{17-19}$, gastrointestinal, piel, linfomas no Hodgkin, vías biliares y cerebro ${ }^{20-22}$. Los metabolitos del PCB's (dioxinas) inducen la transcripción de genes específicos como Ahr y Cyt-P450, CyP1A1, CyP1A2, CyP1B1 que codifican proteínas particulares del metabolismo de xenobióticos, principalmente, la enzima citocromo P-450 1A1 que promueve la generación de productos tóxicos ${ }^{23}$. Asimismo, regula la expresión de genes relacionados con la síntesis de: aldehído deshidrogenasa citosólica, glucuroniltransferasa y la glutationreductasa ${ }^{24}$. El efecto cancerígeno se debe a la afinidad de las dioxinas por el receptor arilhidrocarburo (gen $A h r$ ) que se halla en todos los tejidos ${ }^{25,26}$.

Algunos estudios, en la población general, han reportado que las concentraciones séricas de estos compuestos organoclorados se correlacionan positivamente con la edad ${ }^{27,28}$ pero otros, que los niveles promedio están disminuyendo ${ }^{29,30}$.

El objetivo de éste trabajo fue la búsqueda en sangre de niveles tóxicos de PCB's y sus efectos a nivel orgánico en los trabajadores con mayor exposición de una empresa eléctrica de Barranquilla.

\section{Metodología}

Estudio descriptivo transversal realizado en una muestra de la población de trabajadores de una empresa del sector eléctrico de Barranquilla, muestra constituida por 30 personas expuestas al PCB's y 31 personas no expuestas. Los trabajadores participantes fueron voluntarios, escogidos bajo muestreo no probabilístico por cuotas donde se tomaron dos grupos que difieren en la exposición, con edades entre 18 y 65 años, cuyo criterio de inclusión fue que no presentaran patologías de base y que firmaran el consentimiento informado. Al inicio del estudio se realizó una encuesta para obtener la información requerida en la selección del personal. Así, en el grupo de expuestos al PCB's, trabajadores del servicio de mantenimiento de equipos y redes eléctricas, todos del género masculino, se tuvo en cuenta el tiempo de exposición, los resultados de laboratorio, signos y síntomas. Para el grupo de no expuestos, se tomó personal administrativo de la empresa, de ellas, $49.1 \%(n=13)$ del género femenino, con oficio no relacionado con el agente en estudio y que, además, fueron catalogados por análisis clínico como personas sanas (Figura 2).

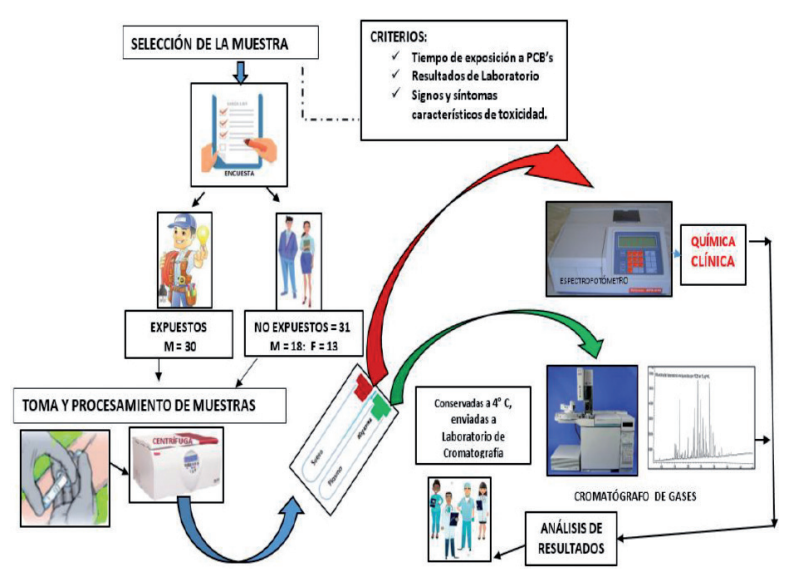

Figura 2. Esquema del diseño metodológico.

A cada paciente, se le extrajo $10 \mathrm{ml}$ de sangre venosa, la cual se distribuyó en dos tubos Vacutainer, uno con heparina y el otro, limpio y seco. Las muestras de plasma obtenidas fueron almacenadas a $4^{\circ} \mathrm{C}$ y enviadas al Laboratorio de Cromatografía de la Universidad Industrial de Santander (UIS, Bucaramanga) para el análisis de bifenilos policlorados. A las muestras de suero se les practicó, a través de la EPS vinculada con la empresa, los exámenes de laboratorio clínico por métodos enzimáticos colorimétricos: glicemia por el método glucosa oxidasa/peroxidasa; colesterol total, utilizando el método colesterol esterasa/oxidasa; 
colesterol de alta densidad (HDLc) por precipitación con polietilenglicol a $\mathrm{pH} \mathrm{10;} \mathrm{triglicéridos,} \mathrm{el} \mathrm{método}$ glicerolfosfato oxidasa/peroxidasa; bilirrubina total, método de Walter Gerarde, el nitrógeno ureico (BUN), por la reacción de Berthelot y creatinina, Jaffé modificado cinético; la medición de la actividad enzimática de: fosfatasa alcalina aplicando un método cinético optimizado, las Transaminasas GlutamicoOxalacética (GOT), Transaminasas Glutamico-Pirúvica (GPT) y Gamma-GlutamilTranspeptidasa (GGT), el método UV cinético; la hormona estimulante de la tiroides (TSH), $\mathrm{T}_{3}$ y $\mathrm{T}_{4}$ por inmunoanálisis. Además, se les practicó un hemograma para observar alteraciones de elementos formes sanguíneos.

Para el tratamiento de las muestras, a cada una se le hizo test de ELISA para VIH.

En la determinación de bifenilos policlorados en plasma se aplicó el método propuesto por Atuma ${ }^{31}$. El análisis cromatográfico se efectuó en cromatógrafo de gases (GC) HP 6890 Serie PLUS (Hewlett-Packard, Palo Alto, California, EE.UU.), dotado con Detector de Captura de Electrones (ECD) y columna DB-5 [5\%-fenil-poli (metilsiloxano), $30 \mathrm{~m}$ x $0.25 \mathrm{~mm} \times 0.25$ $\mu \mathrm{m}]$. La inyección se realizó en modo splitless $\left(\mathrm{V}_{\text {iny }}=1\right.$ $\mu \mathrm{L})$. Se utilizó como material de referencia las mezclas certificadas de Aroclores 1016, 1221, 1232, 1242, 1248, 1254 y 1260 en n-hexano (AccuStandard Inc., 125 Market Street, New Haven, CT 06513, EE.UU). Para el método empleado se registró, el nivel mínimo de cuantificación ( $\mathrm{NMC}=2 \mathrm{NMD}), 8 \mu \mathrm{g} / \mathrm{L}$ y el nivel mínimo de detección (NMD), $4 \mu \mathrm{g} / \mathrm{L}$ del detector de captura de electrones (ECD), de los PCB's (Aroclores) totales. Se obtuvieron los cromatogramas por GC/ECD de la mezcla del material certificado de PCB's y de los extractos obtenidos por extracción liquido-liquido continua de las muestras de plasma.

Los valores críticos de concentración en suero para el riesgo de efectos adversos sobre la salud fueron establecidos por la Human Biomonitoring Commission Values (HBM), a partir de la suma de los bifenilos policlorados 138, 153 y 180: HBMI = 3,5 $\mu \mathrm{g} / \mathrm{L}$ (por debajo de este valor no hay riesgo) y HBMII $=7,0 \mu \mathrm{g} / \mathrm{L}$ (para valores iguales o mayores, si hay riesgo). Las concentraciones séricas entre estos dos valores indican riesgo de una fuente de exposición potencial, a la cual debe reducírsele sus efectos ${ }^{32}$

Se utilizó el software Minitab 15, $(\alpha=0.05)$ en las pruebas Chi-Cuadrado y Exacta de Fisher para verificar la asociación entre la exposición al PCB's y la concentración de diferentes biomarcadores en la sangre de los trabajadores; también, se utilizó la V-CUADRADA DE CRAMER como indicador de la fuerza de asociación entre variables, rango $[0,1]$, a fin de detectar posibles afectaciones del PCB's en la salud de estas personas.

El estudio fue aprobado por el comité de ética de la Universidad Libre.

\section{Resultados}

La edad predominante $(63.3 \%)$ entre los trabajadores expuestos a PCB's fue de 46 a 55 años, y entre los no expuestos, el grupo de 36 a 45 años presentó la mayor frecuencia (48.4\%). En los no expuestos la edad promedio fue de 39.3 años, población más joven debido a su cargo de oficinas o de servicios técnicos no expuestos al PCB's.

El 90\% $(\mathrm{n}=27)$ del grupo de los expuestos tenían el mayor tiempo, más de 15 años, de estar laborando en la empresa; mientras que, sólo ocho trabajadores (26.6\%) de los no expuestos se hallaron en este grupo. El 46.6\% de los no expuestos tenían 1 a 5 años de labores en esta empresa (Figura 3).

El análisis de las encuestas reveló que el 100\% de los trabajadores expuestos laboraban turnos de ocho horas diarias; el 56\% de éstos, trabajaban tres horas de taller en mantenimiento de equipos eléctricos al interior de la empresa y las horas restantes, en la reparación e instalación de redes eléctricas en el exterior. Sólo el 10\% de los trabajadores expuestos tenía contacto directo con sustancias líquidas provenientes de transformadores (aceite dieléctrico) y 90\% de ellos, ocasionalmente.

Respecto a la protección individual, el $100 \%$ de los trabajadores participantes en la investigación afirmaron que la empresa les proporcionaba medidas de protección laboral como uniforme completo, gafas y guantes. Todos hacen uso de éstas, pero con diferente frecuencia: el 93\% usa siempre las medidas de protección laboral, mientras tanto, el $7 \%$ algunas veces.

El 95\% de trabajadores expuestos dieron respuesta negativa a problemas de salud relacionados con el trabajo, presentando buen estado de salud en el momento del examen médico.

La Tabla 1, muestra el número de trabajadores de cada grupo, expuestos y no expuestos, que revelaron cifras de algunos biomarcadores en sangre fuera de los valores normales esperados. 


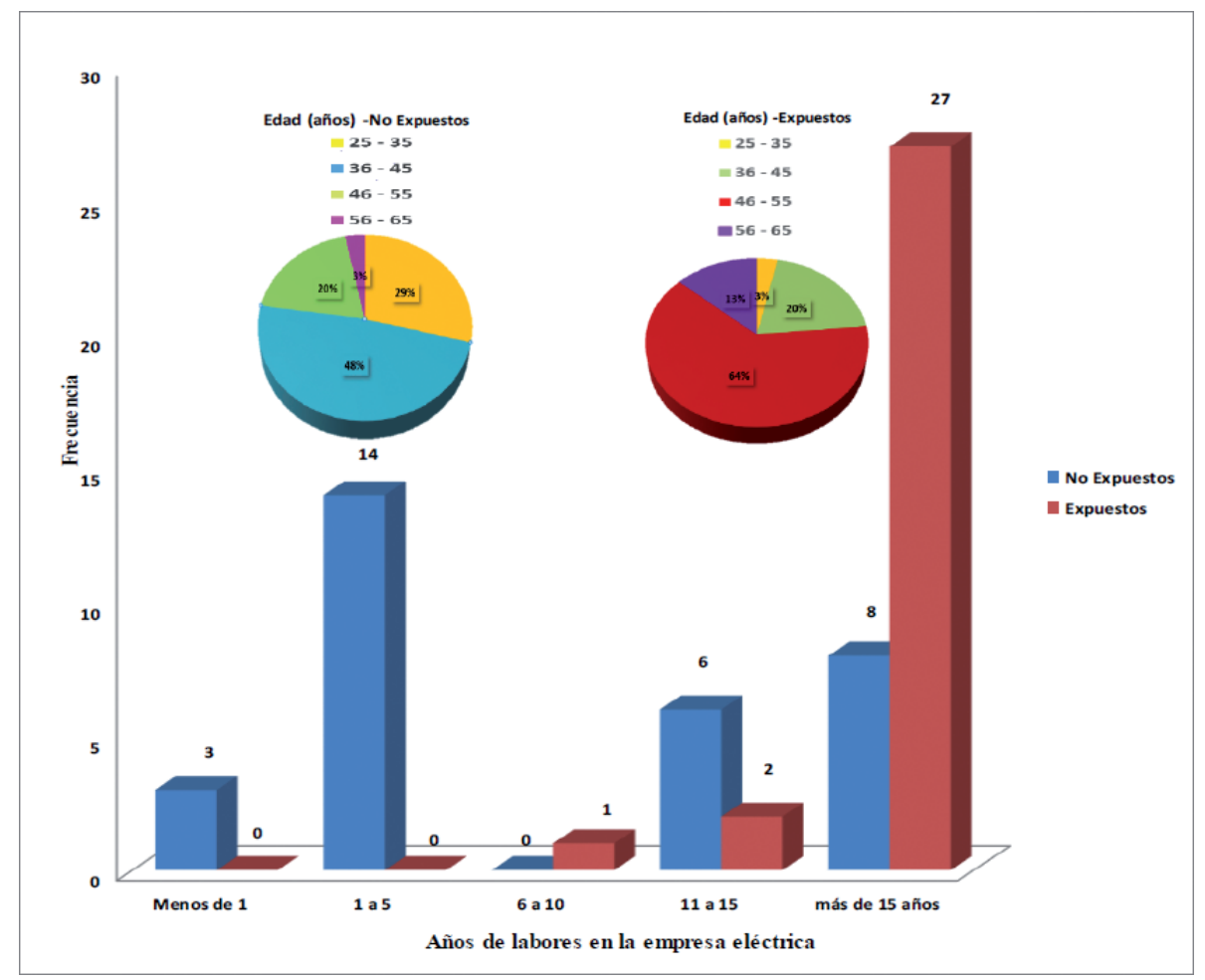

Figura 3. Distribución de frecuencia según edad y tiempo laborando en los talleres de la empresa eléctrica, personal expuesto y no expuesto al PCB's. Barranquilla.

Metabolismo: El 86.7 \% de los trabajadores expuestos se diagnosticaron como normoglicémicos. Solo el $10 \%$ de ellos, tuvieron glicemias $>100 \mathrm{y}<126 \mathrm{mg} /$ $\mathrm{dL}$, ameritando una evaluación de los antecedentes personales y familiares de diabetes y estilos de vida de cada uno, con el fin de evaluar inicio de diabetes tipo 2 y posibles variables de riesgo. Ningún trabajador de los no expuestos al PCB's presentó hiperglicemia; probablemente, los trabajadores expuestos tienen mayor exposición a los carbohidratos debido a su labor que demanda mucha energía, lo que los hace ingerir alimentos no balanceados en sus lugares de trabajo, generalmente, en las calles de la ciudad. Asimismo, no se encontró ningún valor de glicemia $\geq 126 \mathrm{mg} / \mathrm{dL}$, entre ambos grupos.

El $63.3 \%$ de los trabajadores expuestos y $74 \%$ no expuestos tuvieron una colesterolemia $<200 \mathrm{mg} / \mathrm{dL}$ (valor normal). Ninguno de los evaluados no expuestos mostró cifras superiores a $240 \mathrm{mg} / \mathrm{dL}$, mientras que si se observó en el $6 \%$ de los expuestos.

El 100\% de los trabajadores expuestos y no expuestos evaluados presentaron niveles de colesterol HDL $>35 \mathrm{mg} / \mathrm{dL}$, el cual es el parámetro, lo que está de acuerdo con las cifras de colesterol total obtenidas. Según valores normales del método utilizado, sólo tres trabajadores de los expuestos (10\%) y ocho de los no expuestos $(25.8 \%)$, tuvieron resultados inferiores a 40 $\mathrm{mg} / \mathrm{dL}$, pero mayores a $35 \mathrm{mg} / \mathrm{dL}$.

Cifras menores a $100 \mathrm{mg} / \mathrm{dL}$ de LDL colesterol se encontraron en $36.7 \%$ y $38.7 \%$ para expuestos y no expuestos, respectivamente; entre tanto, presentaron cifras superiores a $100 \mathrm{mg} / \mathrm{dL}$, el $63.3 \%$ de los expuestos y $51.3 \%$ de los no expuestos.

En $36.7 \%$ de los participantes expuestos y $38.7 \%$ de los no expuestos se observaron valores superiores a 30 $\mathrm{mg} / \mathrm{dl}$ de VLDL colesterol, constituyendo un factor de riesgo importante para ateroesclerosis.

El $40.0 \%$ de evaluados expuestos presentó niveles de triglicéridos $>150 \mathrm{mg} / \mathrm{dl}$, con un porcentaje similar, $41.9 \%$, los no expuestos; valores $>500 \mathrm{mg} / \mathrm{dl}$ fueron hallados en el 3.3\% de los expuestos; mientras que, entre los no expuestos no hubo ningún caso. Valores entre los expuestos que ameritan un programa de vigilancia epidemiológica para riesgo cardiovascular.

Sistema renal: ningún trabajador presentó afección renal. El 100\% de los trabajadores evaluados, expuestos y no expuestos, presentaron cifras de creatinina y de nitrógeno ureico (BUN) dentro del rango normal. 
Tabla 1. Distribución de la frecuencia según resultados de glicemia, perfil lipídico, nitrógeno ureico y creatininemia en trabajadores del sector eléctrico, expuestos y no expuestos al PCB's, Barranquilla.

\begin{tabular}{|c|c|c|c|c|}
\hline & $\begin{array}{l}\text { Expuestos } \\
\text { (n) }\end{array}$ & $\%$ & $\begin{array}{l}\text { No expuestos } \\
\text { (n) }\end{array}$ & $\%$ \\
\hline \multicolumn{5}{|l|}{ Glicemia (mg/dL) } \\
\hline$<70$ & 1 & 3.3 & 1 & 3.2 \\
\hline$\geq 70$ a 100 & 26 & 86.7 & 30 & 96.8 \\
\hline$>100 \mathrm{y}<126$ & 3 & 10.0 & 0 & 0 \\
\hline Total & 30 & 100 & 31 & 100 \\
\hline \multicolumn{5}{|c|}{ Colesterol total (mg/dL) } \\
\hline Normal $<200$ & 19 & 63.3 & 23 & 74.2 \\
\hline Medio 200 a 240 & 11 & 37.7 & 6 & 19.3 \\
\hline Alto $>240$ & 0 & 0.0 & 2 & 6.5 \\
\hline Total & 30 & 100 & 31 & 100 \\
\hline \multicolumn{5}{|c|}{ Colesterol HDL (mg/dL) } \\
\hline \multicolumn{5}{|l|}{ Normal, 40 a 60} \\
\hline$<40$ & 3 & 10.0 & 8 & 25.8 \\
\hline$>40$ & 27 & 90.0 & 23 & 74.2 \\
\hline Total & 30 & 100 & 31 & 100 \\
\hline \multicolumn{5}{|c|}{ Colesterol LDL (mg/dL) } \\
\hline Normal $<100$ & 11 & 36.7 & 12 & 38.7 \\
\hline Medio 100 a 160 & 12 & 40.0 & 15 & 48.4 \\
\hline Alto $>160$ & 7 & 23.3 & 4 & 12.9 \\
\hline Total & 30 & 100 & 31 & 100 \\
\hline \multicolumn{5}{|c|}{ Colesterol VLDL (mg/dL) } \\
\hline Normal, 2 - 30 & 19 & 63.3 & 19 & 61.3 \\
\hline Alto $>30$ & 11 & 36.7 & 12 & 38.7 \\
\hline Total & 30 & 100 & 31 & 100 \\
\hline \multicolumn{5}{|l|}{ Triglicéridos (mg/dL) } \\
\hline Normal $<150$ & 17 & 56.7 & 18 & 58.1 \\
\hline Medio $\geq 150$ a 500 & 12 & 40.0 & 13 & 41.9 \\
\hline Alto $>500$ & 1 & 3.3 & 0 & 0.0 \\
\hline Total & 30 & 100 & 31 & 100 \\
\hline \multicolumn{5}{|c|}{ Nitrógeno ureico, BUN (mg/dL) } \\
\hline \multicolumn{5}{|c|}{ Normal, 6 a $20 \mathrm{mg} / \mathrm{dL}$} \\
\hline$<6$ & 30 & 100.0 & 31 & 100.0 \\
\hline Total & 30 & 100 & 31 & 100 \\
\hline \multicolumn{5}{|l|}{ Creatinina (mg/dL) } \\
\hline \multicolumn{5}{|c|}{ Normal hasta $1.3 \mathrm{mg} / \mathrm{dL}$} \\
\hline$<1.3$ & 30 & 100.0 & 31 & 100.0 \\
\hline Total & 30 & 100 & 31 & 100 \\
\hline
\end{tabular}

Fuente: Datos del grupo investigador.

Sistema hepático: Cabe resaltar que se obtuvieron valores normales para cada una de las enzimas examinadas: transaminasas pirúvicas, 0 a $40 \mathrm{UI} / \mathrm{L}$, y oxaloacética, 0 a $41 \mathrm{UI} / \mathrm{L}$, la fosfatasa alcalina, 60-300 UI/L, excepto la GGPT, que resultó por debajo del rango normal, 8-61 UI/L, en el $90 \%$ y $93.5 \%$ de los trabajadores expuestos y no expuestos, respectivamente, demostrando que los participantes del estudio, quienes son una fiel representación de la población, no presentan afección hepática.
Sistema endocrino: las pruebas tiroideas TSH, $\mathrm{T}_{3}$ y $\mathrm{T}_{4}$ de los trabajadores evaluados (100\%), expuestos y no expuestos se hallaron dentro de límites normales.

Los resultados del hemograma, de expuestos y no expuestos, fueron normales. El 100\% de los trabajadores expuestos y no expuestos resultaron VIH negativo para el test de ELISA. 
El $100 \%$ de los trabajadores presentaron cifras de PCB's en plasma inferiores al valor límite mínimo de tolerancia, para permitir daño a la salud.
Los datos de los exámenes bioquímicos y detección de PCB's en sangre, detallados en las tablas antes señaladas, fueron evaluados estadísticamente, estableciéndose la Tabla 2, con el resultado de la comparación de estos datos.

Tabla 2. Análisis de asociación entre la exposición al PCB's y el nivel de los biomarcadores en sangre de trabajadores expuestos y no expuestos, de una empresa del sector eléctrico de Barranquilla.

\begin{tabular}{|c|c|c|c|c|c|c|c|c|c|}
\hline \multirow{2}{*}{ Biomarcadores } & \multirow{2}{*}{ Resultados } & \multicolumn{2}{|c|}{ EXPUESTOS } & \multicolumn{2}{|c|}{ NO EXPUESTOS } & \multirow{2}{*}{$\begin{array}{c}\begin{array}{c}\text { Chi- } \\
\text { cuadrado }\end{array} \\
\text { (p-Value) } \\
\end{array}$} & \multirow{2}{*}{$\begin{array}{c}\begin{array}{c}\text { Prueba } \\
\text { exacta } \\
\text { Fisher }\end{array} \\
\text { (p-Value) }\end{array}$} & \multirow{2}{*}{$\begin{array}{l}\text { V-Cuadrada } \\
\text { de Cramer }\end{array}$} & \multirow{2}{*}{$\mathbf{p}$} \\
\hline & & Frecuencia & $\%$ & Frecuencia & $\%$ & & & & \\
\hline \multirow[t]{2}{*}{ Glicemia } & $>100 \mathrm{mg} / \mathrm{dL}$ & 3 & 10.0 & 0 & 0.0 & 0,094 & 0.11 & 0,046 & NS** \\
\hline & $200-240$ & & & & & & & & NS \\
\hline Colesterol Total & $\mathrm{mg} / \mathrm{dL}$ & 11 & 36.7 & 6 & 19.0 & 0.315 & 0.301 & 0.038 & \\
\hline Colesterol HDL & $<40 \mathrm{mg} / \mathrm{dL}$ & 3 & 10.0 & 8 & 25.8 & 0.598 & 0.792 & 0.0046 & NS \\
\hline Colesterol LDL & $>160 \mathrm{mg} / \mathrm{dL}$ & 7 & 23.3 & 4 & 12.9 & 0.602 & 0.599 & 0.017 & NS \\
\hline Colesterol & & & & & & & & & NS \\
\hline VLDL & $>30 \mathrm{mg} / \mathrm{dL}$ & 11 & 36.7 & 12 & 38.7 & 1.0 & 1.0 & 0.00 & \\
\hline Triglicéridos & $>150 \mathrm{mg} / \mathrm{dL}$ & 13 & 43.3 & 13 & 41.9 & 0.914 & 1.0 & 0.0002 & NS \\
\hline STGO* & $>30 \mathrm{UI} / \mathrm{L}$ & 0 & 0.0 & 0 & 0.0 & & & & \\
\hline SGPT* & $>41 \mathrm{UI} / \mathrm{L}$ & 0 & 0.0 & 0 & 0.0 & & & & \\
\hline GGPT & $>50 \mathrm{UI} / \mathrm{L}$ & 3 & 10.8 & 2 & 8.2 & 0.148 & 0.18 & 0.035 & NS \\
\hline BUN* & $>6 \mathrm{mg} / \mathrm{dL}$ & 0 & 0.0 & 0 & 0.0 & & 0.237 & 0.036 & NS \\
\hline Creatinina* & $>1,3 \mathrm{mg} / \mathrm{dL}$ & 0 & 0.0 & 0 & 0.0 & & & & \\
\hline \multicolumn{10}{|l|}{ Fosfatasa } \\
\hline Alcalina* & > $300 \mathrm{UI} / \mathrm{L}$ & 0 & 0.0 & 0 & 0.0 & 0.149 & 0.194 & 0.03 & NS \\
\hline Bilirrubina total* & $>1.9 \mathrm{mg} / \mathrm{dL}$ & 0 & 0.0 & 0 & 0.0 & & & & \\
\hline PCB's + & $\geq 4 \mu \mathrm{g} / \mathrm{L}$ & 0 & 0.0 & 0 & 0.0 & & & & \\
\hline
\end{tabular}

Fuente: datos del grupo investigador.

* Ninguno $(0.0 \%)$ de los trabajadores evaluados, expuestos y no expuestos, presentaron cifras anormales.

**NS: No fueron estadísticamente significativas.

+ Todos los trabajadores presentaron resultados inferiores al "Nivel mínimo de detección (NMD): $4 \mu \mathrm{g} / \mathrm{L}$ " de la técnica cromatográfica de gas utilizada.

\section{Discusión}

La biomonitorización ${ }^{33}$ o cuantificación de las concentraciones de sustancias o metabolitos tóxicos en muestras de sangre y fracciones, orina, tejidos y fluidos como pelos, uñas, leche materna, entre otros, pueden demostrar si los niveles de PCB's son mayores que lo normal, pero no pueden determinar cuándo o por cuanto tiempo estuvo expuesto o si afectará la salud, de acuerdo con la Agencia para Sustancias Tóxicas y el Registro de Enfermedades(ASTDR) ${ }^{5}$, es decir, el efecto de los PCB's sobre la salud es difícil de establecer como sucede con los Compuestos Orgánicos Persistentes $(\mathrm{COPs})^{34}$, en específico, las dioxinas ${ }^{35}$.
En esta investigación no se demostró daño a la salud en la población ocupacionalmente expuesta, lo cual concuerda con estudios realizados anteriormente a nivel mundial por otros grupos de investigadores:

El NIOSH (Instituto Nacional para la Seguridad Ocupacional y Salud) estudió trabajadores de mantenimiento expuestos a los PCB's en dos compañías eléctricas. Las concentraciones de PCB's en sangre eran mucho más elevadas en esos trabajadores que en el nivel nacional de fondo (12 a 298 partes por mil millones [ppmm] versus 10 a 20 ppmm. Los trabajadores no demostraron tener ningún efecto sobre su salud, ni siquiera cloroacné ${ }^{36}$. 
Lawton, et al. estudiaron la salud de 194 trabajadores fuertemente expuestos al PCB's durante un promedio de 15 años, con algunos casos hasta 35 años. El primer informe de trabajadores seguidos hasta 1976 no mostró efectos adversos. Una reevaluación en 1979 tampoco produjo evidencias de efectos adversos sobre la salud de estos trabajadores ${ }^{37}$.

Del mismo modo, no hay evidencia concluyente sobre que los niveles plasmáticos de PCB's en la población de trabajadores de la empresa eléctrica, no expuestos y expuestos ocupacionalmente, dieran por resultado efectos agudos, probablemente, debido a que la sensibilidad usada en el método de cromatografía de gas no fue lo suficiente para ser detectados y proporcionar datos que sirvieren de base para la comparación.

En esta población de expuestos no se observaron efectos adversos al PCB's. Los valores normales de los exámenes clínicos evaluados reflejan un buen estado de salud.

Lo anterior concuerda con estudios realizados ${ }^{5}$, en donde concluyeron que los PCB's están disminuyendo en sangre humana y animal, probablemente, por la disminución en su uso, las recomendaciones de uso por parte de las autoridades competentes, y el cuidado durante la manipulación por parte de las empresas que los utilizan con fines industriales (Salud Ocupacional).

\section{Conclusiones y recomendaciones}

En las muestras de plasma, de los trabajadores expuestos y no expuestos que participaron en el estudio, no se detectaron PCB's por encima del nivel mínimo de detección del método empleado, decir, los resultados fueron inferiores a $4 \mu \mathrm{g} / \mathrm{L}$.

En general, los resultados obtenidos de las evaluaciones clínicas muestran que no existen evidencias para cualquier daño orgánico en todos los trabajadores expuestos y no expuestos a PCB's, presentando buen estado de salud al momento del examen.

La edad promedio del grupo de expuestos fue de 48.9 años ( \pm 6.4 años, Desviación Estándar), esto se traduce en población con mayor exposición por la edad, permitiendo una mejor comparación entre exposición y compromiso orgánico.

No se encontró diferencias estadísticamente significativas entre los resultados obtenidos de las evaluaciones clínicas entre trabajadores expuestos y no expuestos al PCB's.

La Salud Ocupacional y la Salud Pública son un pilar fundamental para que la salud del trabajador se mantenga y sea productivo, se sienta útil a la sociedad, y sea saludable para disfrutar de las actividades cotidianas.

Para optimar los análisis de riesgo para la salud se sugiere, continuar con evaluaciones periódicas a los trabajadores ocupacionalmente expuestos a los PCB's, la capacitación permanente para concientizarlos sobre el uso obligatorio de elementos de protección personal que eviten mayor contacto, bloqueando las vías de entrada del PCB's al organismo y realizar ensayos de: polimorfismo genético, que afecta la capacidad de un organismo para biotransformar un xenobiótico, de genotoxicidad y de citotoxicidad.

\section{Agradecimientos}

A la Universidad Libre Seccional Barranquilla y Electricaribe S.A. por los fondos para la realización del presente estudio.

\section{Referencias}

1. Organización Mundial de la Salud (OMS): Las dioxinas y sus efectos en la salud humana; 2014.

2. Organización Mundial de la Salud (OMS): "Polychlorinated biphenyls: Human health aspects. Concise international chemical assesment document 55". Informe de consenso científico. Programa Internacional de Seguridad de las Sustancias Químicas (IPCS); 2003.

3. Moreno Grau MA, García Brage A. Toxicología Ambiental: Evaluación de riesgo para la salud humana. Editorial Mc Graw Hill. 2003, pp. 305.

4. Robin MM. The World According to Monsanto: Pollution, Corruption, and the Control of the World's Food Supply, New Press; 2010.

5. Agencia para Sustancias Tóxicas y el Registro de Enfermedades (ATSDR). Bifenilos Policlorados (PCB's).

6. Programa de las Naciones Unidas para el Medio Ambiente (PNUMA). Bifenilos policlorados PCB's.

7. Eskenazi B, Chevrier J, Goldman Rosas L, Anderson HA, Bornman M, Bouwman H, et al. The pine river statement: human health consequences of DDT use. Environ Health Perspect. 2009; 117(9): 1359-1367. doi: 10.1289/ehp.11748. 
8. Colombia, Congreso de la República. Ley 253 de 1996. mediante la cual Colombia adhiere al Convenio de Basilea. Bogotá D.C. 1996.

9. Colombia, Congreso de la República. Ley 1196 del 5 de julio del 2008. Por medio de la cual se aprueba el "Convenio de Estocolmo sobre Contaminantes Orgánicos Persistentes”. Diario. Bogotá, D.C. Oficial No. 47.011. P. 1-77.

10. Instituto Nacional de Salud Pública, Grupo factores de riesgo ambiental. Vigilancia y análisis del riesgo en salud pública. Protocolo de vigilancia en salud pública. Intoxicaciones por sustancias químicas. Ministerio de Salud y Protección Social de Colombia (Min Salud).2016, Versión 02: 1-75.

11. Alcarraz M, Flores A, León J. Aislamiento primario de microorganismos lipolíticos a partir de efluentes de curtiembres. Laboratorio de Microbiol Amb Biotecnol; 2005.

12. Carrillo Cruz A, Moreno Figueredo G, Lara Osorio M. Toxicología de las dioxinas y su impacto en la salud humana. Rev Med Vet. 2010; 19.

1. 13. Miller-Pérez C, Sánchez-Islas E, MucioRamírez S. Los contaminantes ambientales bifenilos policlorinados (PCB) y sus efectos sobre el sistema nervioso y la salud. Salud Ment. 2009; 32(4): 335-346.

14. Ruiz P, Faroon O, Moudgal CJ, Hansen H, De Rosa CT, Mumtaz M. Prediction of the health effects of polychlorinated biphenyls (PCB's) and their metabolites using quantitative structure-activity relationship (QSAR). Toxicol Lett. 2008; 181(1): 53-65. doi: 10.1016/j.toxlet.2008.06.87015. Organización Mundial de la Salud (OMS). Centro de Prensa. Las dioxinas y sus efectos en la salud humana; 2014.

16. Tharappel JC, Lee EY, Robertson LW, Spear BT, Glauert HP. Regulation of cell proliferation, apoptosis, and transcription factor activities during the promotion of liver carcinogenesis by polychlorinated biphenyls. Toxicol Appl Pharmacol. 2002; 179(3): 172-184. doi: 10.1006/ taap.2001.9360.

17. Luna-Sánchez S, Lobeto Martínez MR, Garay Muñoz JS. Exposición laboral a hidrocarburos clorados y cáncer: revisión de la bibliografía reciente. Med Segur Trab, 2014; 60(235): 406-419. doi:http://dx.doi.org/10.4321/S0465546X2014000200012.

18. Alguacil J, Porta M, Malats N, Kauppinen T, Kogevinas M, Benavides F, et al. Occupational exposure to organic solvents and K-ras mutations in exocrine pancreatic cáncer. Carcinogenesis. 2002; 23(1): 101-106.
19. Ojajärvi A, Partanen T, Ahlbom A, Boffetta P, Hakulinen T, Jourenkova N, et al. Occupational exposure and pancreatic cancer: a meta-analysis. Occup. Environ med. 2000; 57(5): 316-324.

20. Oakley GG, Devanaboyina U, Robertson LW, Gupta RC. Oxidative DNA damage induced by activation of polychlorinated biphenyls (PCBs): implications for PCB-induced oxidative stress in breast cancer. Chem Res Toxicol. 1996; 9(8): 1285-1292. doi: 10.1021/tx960103o.

21. Laden F, Ishibe N, Hankinson SE, Wolff MS, Gertig DM, Hunter DJ, et al. Polychlorinated biphenyls, cytochrome P-450 1A1, and breast cancer risk in the Nurses' Health Study. Cancer Epidemiol Biomarkers Prev. 2002; 11(12): 1560-1565.

22.Zhang Y, Wisw JP, Holford TR, Xie H, Boyle P, Zahm SH, et al. Serum polychlorinated biphenyls, cytochrome P-450 1A1 polymorphisms, and risk of breast cancer in Connecticut women. Am J epidemiol 2004; 160(12): 1177-1183. doi: 10.1093/aje/.

23. Carpenter OD. Polychlorinated biphenyls (PCBs): routes of exposure and effects on human health. Rev Environ Health. 2006; 21: 1-23.

24.|Korkalainen M. Structure and expression of principal proteins involved in dioxin signal transduction and potentially in dioxin sensitivity. Publications of the National Public Health Institute, KTL A11/2005.

25. Toumisto J, Pekkanen J, Kiviranta H, Tukiainen E, Vartiainen T, Viluksela, M. Dioxin cáncer riskexample of hormesis. Dose Response. 2005; 3(3): 332-341. doi: 10.2203/dose-response.003.03.004.

26. Argemi E, Cianni N, Porta A. Disruption endocrina: perspectivas ambientales y salud pública. Acta Bioquim Clin Latinoam. 2005; 39(3): 291-300.

27. Patterson DG, Turner WE, Caudill SP, Needham LL. Total TEQ reference range (PCDDs, PCDFs, cPCBs, mono-PCBs) for the US population 20012001. Chemosphere. 2008; 73(Suppl 1): S261-277. doi: 10.1016/j.chemosphere.2007.08.074.

28. Patterson DG, Won Ly, Turner WE, Caudill SP, Dipietro ES, McClure PC, et al. Levels in the U.S. population of those persistent organic pollutans (2003-2004) included in the Stockholm Convention or in other long-range transboundary air pollution agreements. Environ Sci Technol. 2009; 43(4): 12111218. doi: 10.1021/es801966w.

29. Furst P. Dioxins, polyclorinados bifenils and other organohalogen compounds in human milk. Level, correlations, trends and exposure through breastfeeding. Mol Nutr Food Res. 2006; 50(10): 922-933. doi: 10.1002/mnfr.200600008. 
30. Humbert O, Sergeyev O, Altshul L, Korricks SA, Williams PL, Claude E, et al. Temporal trends in suero concentrations of polychlorinated dioxins, furans, and PCBs among adults women living in Chapaevsk, Russia: a longitudinal study from 2000 to 2009. Environ Health. 2011;10: 62. doi: 10.1186/1476-069X-10-62.

31. Atuma S. Method for the determination of PCB congeners and chlorinated pesticides in human blood serum. Enviro Contaminat Toxicol. 1999; 62(1): 8-15. doi: 10.1007/s001289900834.

32. Human Biomonitoring Commission (HBM Commission). Human biomonitoring (HBM) is an important tool in environmental medicine to asses and evaluate the level of internal exposure of the general population, population groups and individuals to environmental toxins.

33. Ibarluzea J, Aurrekoetxea J, Porta M, Sunyer J, Ballester F. La biomonitorización de sustancias tóxicas en muestras biológicas de población general. Gaceta Sanit. 2016; 30(Suppl 1) : 45-54. doi: https://doi.org/10.1016/j.gaceta.2016.02.012.

34. Garcia-Cuan A, Porto DR, Lalinde MJS. Panorama actual de los contaminantes orgánicos persistentes. Biociencias. 2012, 7(1): 81-88.

35. Loayza J, Silva M, Arce G, Casafranca A. Gestión integral de residuos de bifenilos policlorados PCB (Aspectos generales y ciclo de vida). Rev Per Quim Ing Quím. 2015; 18(2): 31-39.

36. Smith AB. Draft report: Cross-Sectional Survey of a Group of Workers Occupationally Exposed to Polychlorinated Biphenyls (PCB) at an Electrical Equipment Manufacturing Plant. Br J Ind Med. 1982; 39(4): 361-369.

37. Lawton RE, Ross MR, Feingold J, Brown JF. Effects of PCB exposure on biochemical and hematological findings in capacitor workers. Envir Health Perspect. 1985; 60: 165-184. doi: 10.1289/ ehp.8560165. 